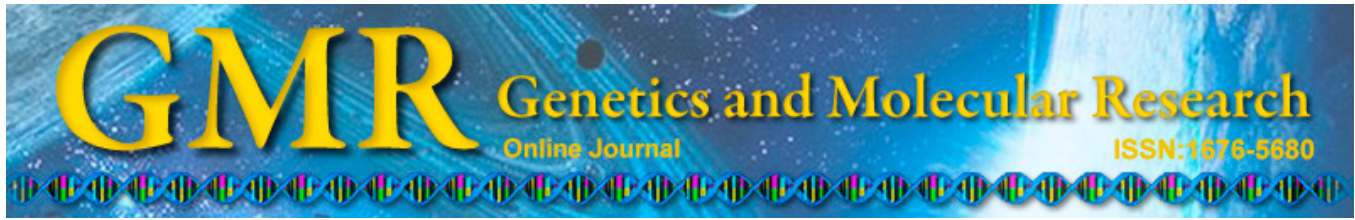

\title{
Quantitative changes in mitochondrial DNA copy number in various tissues of pigs during growth
} \author{
X.W. Li \\ Institute of Animal Genetics and Breeding, \\ College of Animal Science and Technology, \\ Sichuan Agricultural University, Ya'an, Sichuan, China \\ *These authors contributed equally to this study. \\ Corresponding author: X.W. Li \\ E-mail: xuewei.li@sicau.edu.cn
}

Y.M. Xie*, L. Jin*, X.J. Chen, M.N. He, Y. Wang, R. Liu, M.Z. Li and

Genet. Mol. Res. 14 (1): 1662-1670 (2015)

Received February 12, 2014

Accepted August 21, 2014

Published March 6, 2015

DOI http://dx.doi.org/10.4238/2015.March.6.12

\begin{abstract}
Mitochondrial DNA (mtDNA) content is dependent on the energy requirements of tissues. To date, no comprehensive study has been conducted to examine mtDNA copy number variations in pigs. In the current study, quantitative polymerase chain reaction was performed to quantify the mtDNA copy number in 15 pig tissue types at 5 growth stages from embryo to adult. Observable differences in mtDNA content were detected in the tissues, including a 6-fold greater mtDNA content in the heart compared with the lung of 180-day-old samples. mtDNA content in the heart, longissimus dorsi muscle, psoas major muscle, kidney, brain, ovary, and subcutaneous adipose increased with growth. Expression of the replicative mitochondrial helicase (TWINKLE), which regulates mtDNA turnover, was significantly associated with changes in mtDNA copy numbers across tissues during growth $(\mathrm{r}=0.33, \mathrm{P}=0.01)$. We demonstrated that the
\end{abstract}


expression levels of mitochondrial genes were positively correlated with mtDNA copy number.

Key words: Growth stages; Mitochondrial DNA copy number; Gene expression; Pig

\section{INTRODUCTION}

Mitochondria are unique organelles because they contain their own DNA. A somatic mammalian cell contains approximately 1000-10,000 copies of mitochondrial DNA (mtDNA) (Larsson and Gustafsson, 2007). In general, mtDNA is a circular, double-stranded DNA molecule that is approximately $16 \mathrm{~kb}$ in length and encodes 13 protein subunits of the electron transport chain, 2 ribosomal RNAs, and 22 transfer RNAs, translated from its own mRNAs (Larsson and Gustafsson, 2007). The 2 strands of mtDNA exhibit different sedimentation properties in alkaline cesium chloride gradients, and are therefore designated as the heavy and light strands. mtDNA content is frequently used as a marker of mitochondrial density, reflecting the oxidative or ATP-producing capacity of tissues (Hoeks and Schrauwen, 2012). mtDNA copy number has been extensively studied in the tissues of humans (Miller et al., 2003), rats (Moayyeri et al., 2012), and mice (Masuyama et al., 2005), and has been shown to vary widely in different tissues. Studies in humans (Pesce et al., 2001) and mice (Masuyama et al., 2005) demonstrated that mtDNA copy number increased in the heart and skeletal muscle during growth and development.

Sophisticated regulatory mechanisms controlled by nuclear genes regulate mitochondrial DNA replication and transcription (Larsson and Gustafsson, 2007). TWINKLE is the only replicative DNA helicase in the mammalian mitochondria encoded by the nuclear genome. Previous studies have indicated that changes in TWINKLE expression influence mtDNA content. Such changes in mtDNA content may also regulate the expression of mitochondrial genes (Barazzoni et al., 2000, Welle et al., 2000), with an integral role in oxidative phosphorylation.

To date, no systematic study has been conducted to examine mtDNA content in various tissues of pigs during the development process. In this study, we quantitatively analyzed mtDNA content in 15 tissue types at 5 growth stages using quantitative polymerase chain reaction (qPCR) in pigs. We also quantified the mRNA level of TWINKLE and 8 mitochondrial genes in each of the 15 tissues at 5 growth stages to elucidate the relationship between mtDNA content and nuclear gene mtDNA transcripts.

\section{MATERIAL AND METHODS}

\section{Ethics statement}

All animal protocols were approved by the Institutional Animal Care and Use Committee of Sichuan Agricultural University, and all animal work was approved by the appropriate committee.

\section{Animals and tissue collection}

In this study, we collected 15 types of tissues from 3 healthy female Jinhua pigs representing 1 prenatal stage (90 days after insemination, E90) and 4 postnatal stages $(0,30$, and 
180 days and 7 years after birth, referred to as D0, D30, D180, and Y7, respectively). These tissues included the heart, liver, spleen, lung, kidney, brain, ovary, longissimus dorsi muscle (LDM), psoas major muscle (PMM), subcutaneous adipose (SAT), abdominal adipose, mesenteric adipose, greater omentum, retroperitoneal adipose, and intermuscular adipose tissue. Tissues were rapidly dissected and frozen immediately in liquid nitrogen, and stored at $-80^{\circ} \mathrm{C}$ until use.

\section{DNA and RNA extraction}

DNA was isolated from all tissues using the TIANamp MicroDNA Kit (Tiangen, Beijing, China), and total RNA was extracted from frozen adipose tissues using TRIzol (Takara, Shiga, Japan) according to the manufacturer protocol. DNA and RNA concentration and integrity were analyzed by $1 \%$ agarose gel electrophoresis.

\section{Quantification of mtDNA copy number}

Relative amounts of mtDNA and nuclear DNA were determined by qPCR. The mitochondrial genes $A T P 6, C O X 1$, and ND1 were used to quantify mtDNA and nuclear gene. The glucagon gene $(G C G)$, which is highly conserved between species and is present as a single copy in animals (Wang et al., 2012), was used as the single-copy reference gene. The $\mathrm{Ct}$ value of ATP6, COX1, and ND1 versus the Ct value of the single-copy gene was used to reflect the mtDNA amount per haploid cell. The data are reported as means \pm standard deviation. Primer sequences are listed in Table 1.

\begin{tabular}{|c|c|c|}
\hline Gene symbol & Primer sequence ( $5^{\prime}$ to $\left.3^{\prime}\right)$ & Amplicon size (bp) \\
\hline \multirow[t]{2}{*}{ ATP6 } & F: TATTTGCCTCTTTCATTGCCC & 123 \\
\hline & R: GGATCGAGATTGTGCGGTTAT & \\
\hline \multirow[t]{2}{*}{$C O X I$} & F: ACTACTGACAGACCGCAACC & 220 \\
\hline & R: TCCAATGGACATTATGGCTC & \\
\hline \multirow[t]{2}{*}{$C O X 2$} & F: TGAAGACCTCACCTTTGACTC & 193 \\
\hline & R: TCCTGGGATAGCATCTGTTTT & \\
\hline \multirow{2}{*}{ CytB } & F: TCTTCGCCTTTCACTTTATC & 240 \\
\hline & R: GGGTGTAGTTGTCTGGGTCT & \\
\hline \multirow[t]{2}{*}{ ND1 } & F: GCCACATCCTCAATCTCCAT & 99 \\
\hline & R: GATTAGAGGGTAGGGTATTGGTAG & \\
\hline \multirow[t]{2}{*}{ ND2 } & F: GACTAAACCAAACCCAACT & 189 \\
\hline & R: GGGATAGGGATAAAGTTGT & \\
\hline \multirow[t]{2}{*}{ ND3 } & F: CCTCCСТАCTCGTACTAATCG & 210 \\
\hline & R: GATTGTTTGTTTGGGATGCT & \\
\hline \multirow[t]{2}{*}{ ND4 } & F: ACGGACTCCATCTTTGACTG & 217 \\
\hline & R: AGGTCGGTTTGACGTAAGC & \\
\hline \multirow[t]{2}{*}{ ND6 } & F: TGGCTACTGAGATGTACCCT & 205 \\
\hline & R: CCCATAGCTTCCTCACTAAA & \\
\hline \multirow[t]{2}{*}{ TWINKLE } & F: GGAAGGAGGATGATGATAAGG & 211 \\
\hline & R: GGTATGGAGAAGGTAAGAGAGC & \\
\hline \multirow[t]{2}{*}{$G C G^{*}$} & F: GAATCAACACCATCGGTCAAAT & 198 \\
\hline & R: CTCCACCCATAGAATGCCCAGT & \\
\hline \multirow[t]{2}{*}{$P P I A^{*}$} & F: CACAAACGGTTCCCAGTTTT & 171 \\
\hline & R: TGTCCACAGTCAGCAATGGT & \\
\hline \multirow[t]{2}{*}{$R P L 4^{*}$} & F: AGGAGGCTGTTCTGCTTCTG & 185 \\
\hline & R: TCCAGGGATGTTTCTGAAGG & \\
\hline \multirow[t]{2}{*}{$Y W H A Z^{*}$} & F: ATTGGGTCTGGCCCTTAACT & 146 \\
\hline & R: GCGTGCTGTCTTTGTATGACTC & \\
\hline
\end{tabular}

*Reference genes. 
PCR amplification was performed using the CFX-96 detection system (Bio-Rad, Hercules, CA, USA) in $25 \mu \mathrm{L}$ using the SYBR PrimeScript q-PCR Kit (Takara). Each reaction was performed in triplicate and included a negative control (without template).

\section{Measurement of mRNA levels}

All genomic DNA was eliminated from the RNA samples using the PrimeScript RT reagent Kit (Takara), and cDNA synthesis was performed in a $20-\mu \mathrm{L}$ volume. The transcripts of mitochondrial genes, including ATP6, CYTB, COX1, COX2, ND2, ND3, ND4, and ND6, were measured using qPCR with the CFX-96 detection system. Ribosomal protein (RPL4), peptidylprolyl isomerase A (PPIA), and tyrosine 3-monooxygenase/tryptophan 5-monooxygenase activation protein, zeta polypeptide (YWHAZ), were used as reference genes because of their relatively stable expression levels at different ages and in various tissues (Uddin et al., 2011). The expression level of the nuclear gene TWINKLE, which is a determinant of mtDNA levels, was also measured. Detailed information regarding the primers used is listed in Table 1.

\section{Statistical analysis}

The mRNA expression level was quantified using the $2^{-\Delta \Delta \mathrm{Ct}}$ method, and data are reported as means \pm standard deviation. The statistical tests were performed using one-way analysis of variance. We also calculated the Pearson correlation coefficient between mitochondrial genes or TWINKLE expression levels and mtDNA copy number using SigmaPlot 12.3 (Systat Software, Inc., San Jose, CA, USA).

\section{RESULTS AND DISCUSSION}

\section{Different mtDNA content among tissues}

The mtDNA content in various tissues of pigs at different ages was determined by selecting 3 short regions in 3 genes (ATP6, COX1, and ND1) of mtDNA and performing qPCR analysis. The standard curve for each region showed high amplification efficiencies (Figure 1). In all growth stages, the mtDNA copy number showed a significant difference among tissues (Figure 2). For example, the mtDNA copy number in the heart of 180-day-old pigs was 6 -fold higher than in the lung $(\mathrm{P}<0.01)$. The mtDNA content in the heart of 180 -day-old pigs was $\sim 2000$ copies per cell, which was significantly higher than that in other tissues. The higher mtDNA content observed in the heart may be associated with the relatively high energy required for this muscle. We found that the mtDNA content in the skeletal muscles, LDM and PMM, were $~ 1600$ copies per cell, which was slightly lower than in the heart, whereas the adipose tissues showed the lowest mtDNA content ( $\sim 400$ copies per cell). Our results suggest that mtDNA content represents the cell's energy requirements. The heart is one of the most energydemanding tissues in the body, and thus heart cells possess the greatest abundance of mtDNA. Generally, skeletal muscle fibers can be considered to be oxidative (red muscle) or glycolytic (white muscle) types. Oxidative muscle fibers possess a greater number of mitochondria compared to white muscles. Previous studies indicated that PMM is a classic oxidative muscle fiber, while LDM is a glycolytic muscle fiber (Bakala et al., 2013). In the current study, we found that PMM showed higher mtDNA copy numbers compared with LDM. The amount of 
mtDNA in white adipose tissue (retroperitoneal adipose, mesentric adipose, greater omentum, abdominal adipose, and intermuscular adipose tissue) was lower than in the heart and skeletal muscle. This result was as expected considering that the main function of white adipose tissue is to store high-energy molecules (Cinti, 2006). Recently, a new type of adipose tissue, brown adipose tissue, was found to be important for both basal and inducible energy expenditure in the form of thermogenesis (Cypess et al., 2009). This tissue contains an unusually high amount of mitochondria. Thus, the mtDNA content can reflect the energy demands of tissues.

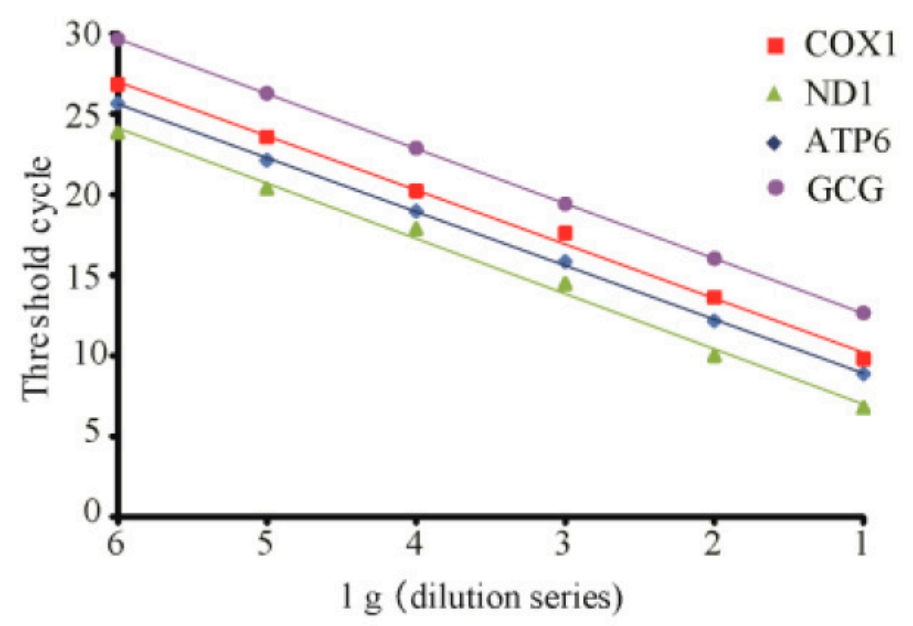

Figure 1. Standard curve for quantitation of mtDNA. Image shows PCR product from serial dilutions. The correlation coefficients were all above 0.99 , and the amplification efficiency was 0.95-0.99.

\section{Growth-associated changes in mtDNA content in different tissues}

We found that mtDNA copy number increased in the heart, PMM, LDM, kidney, brain, ovary, and SAT with growth (Figure 2).

A dramatic increase in mtDNA copy number occurs during the first year of life (Pohjoismäki et al., 2010). In the current study, the mtDNA content in the heart of newborn piglets was nearly 7 -fold lower than in that of adults. Growth-associated increases in mtDNA content in the heart of animals may result from the incessant contractile activity of the heart and the high energy demand required for this process. Additionally, mtDNA copy number also increased gradually with age in PMM and LDM, and mtDNA content increased with muscle development (Pesce et al., 2001). Exercise, particularly aerobic exercise, is known to activate mitochondriogenesis and increases mtDNA levels in skeletal muscle (Lanza et al., 2008). We therefore predicted that the increase in exercise intensity associated with the growth of pigs caused a corresponding increase in mtDNA content. In our study, mtDNA copy number increased during development of SAT $(\mathrm{r}=0.970, \mathrm{P}=0.026)$. A previous study in human white fat cells showed that mtDNA copy number was strongly related to adipocyte lipogenesis (Kaaman et al., 2007); therefore, the increase in mtDNA copy number associated with growth in SAT in this study indicated accumulation of lipids during adipose tissue development. We also observed that the amount of mtDNA significantly decreased during the fifth growth stage in the liver. It has been reported previously that age-related cell loss occurs remarkably in 
some tissues, including the liver (Masuyama et al., 2005), leading to decreased mtDNA levels. Such a reduction in mtDNA content may result in a decline in liver function (Yin et al., 2004).

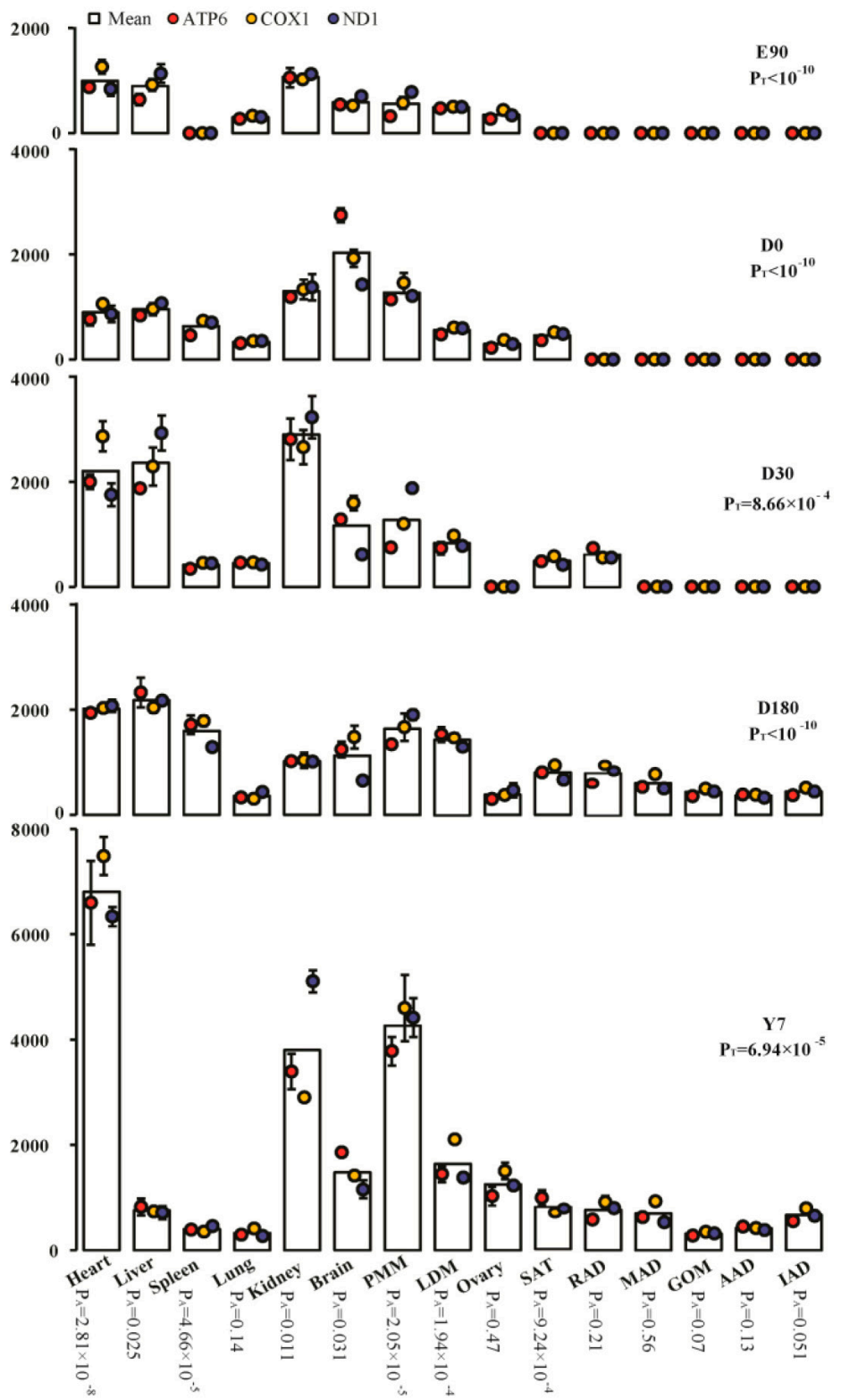

Figure 2. Changes in mtDNA content in various tissues during growth. The bars represent means \pm standard deviation. ' $\mathrm{P}_{\mathrm{A}}$ ' and ' $\mathrm{P}_{\mathrm{T}}$ ' represent the $\mathrm{P}$ value for age and tissue, respectively. $\mathrm{E} 90$ represents embryonic 90 days; D0, D30, D180, and Y7 represent postnatal 0, 30, and 180 days and 7 years. PMM = psoas major muscle; LDM $=$ longissimus dorsi muscle; $\mathrm{SAT}=$ subcutaneous adipose; $\mathrm{RAD}=$ retroperitoneal adipose; $\mathrm{MAD}=$ mesenteric adipose; $\mathrm{GOM}=$ greater omentum; $\mathrm{AAD}=$ abdominal adipose; $\mathrm{IAD}=$ intermuscular adipose. 


\section{Pattern of TWINKLE mRNA expression}

TWINKLE is the only replicative DNA helicase in mammalian mitochondria. Studies examining TWINKLE overexpression and TWINKLE knockout have shown that TWINKLE is an essential mitochondrial helicase required for complete mtDNA replication in the heart and skeletal muscle of mice (Tyynismaa et al., 2004; Kaaman et al., 2007, Milenkovic et al., 2013). The relationship between TWINKLE and mtDNA content during growth was investigated by quantifying the mRNA levels of TWINKLE using qPCR. We found that mtDNA copy number per cell was positively correlated with the expression of TWINKLE $(\mathrm{r}=0.33, \mathrm{P}=0.01)$, as shown in Figure 3. Thus, TWINKLE may be a key regulator of the biogenesis process of mtDNA during pig growth.

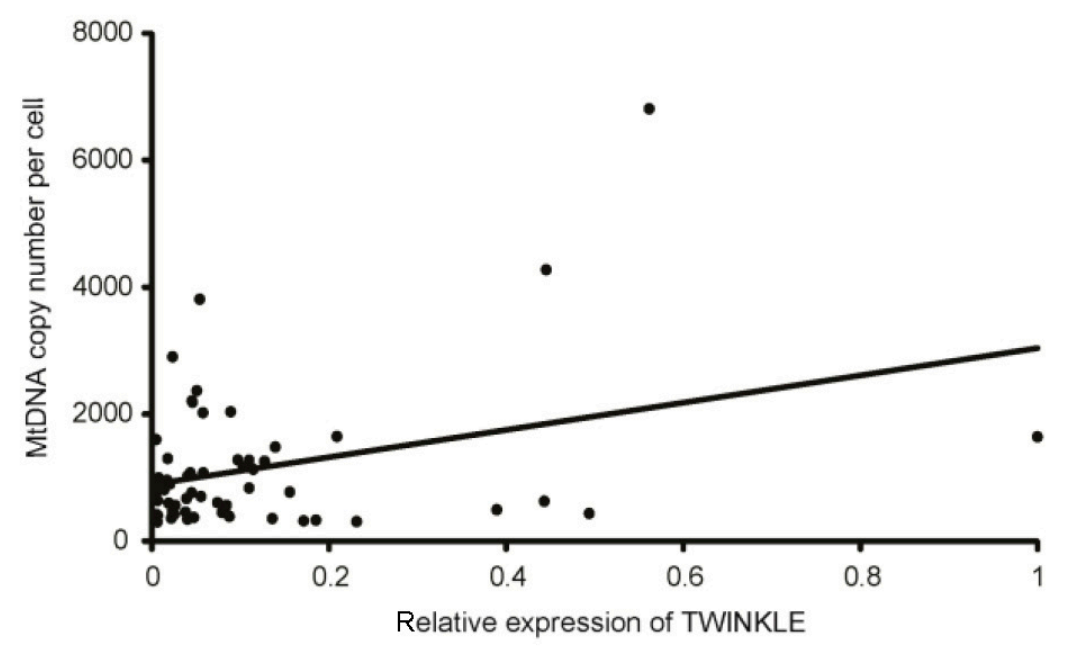

Figure 3. Linear regression analysis to analyze the association between the expression of TWINKLE and mtDNA copy number across samples $(r=0.33, \mathrm{P}=0.011)$.

\section{Expression patterns of mitochondrial genes during growth}

mtDNA-induced changes in mitochondrial gene expression were investigated by quantifying 8 mitochondrial genes selected randomly from both heavy and light strands during growth. As shown, the expression of 7 genes was significantly and positively correlated with mtDNA content (Figure 4). The transcription of mtDNA proceeds through polycistronic precursor RNAs, encompassing all genetic information encoded in each of the specific strands (Larsson and Gustafsson, 2007). These primary transcripts are processed to produce individual mRNA, rRNA, and tRNA molecules (Clayton, 1991), which are thought to be expressed at similar levels. However, we found that each gene was expressed at a different level and hypothesized that this discrepancy was caused by post-transcriptional regulation, such as by miRNAs (Latronico and Condorelli, 2012; Das et al., 2012; Sripada et al., 2012). Hence, mtDNA copy number was only 1 factor influencing mitochondrial function. In addition, oxidative functional changes in mitochondria should be examined, particularly cytochrome $\mathrm{c}$ activity or ATP-producing capacity. 


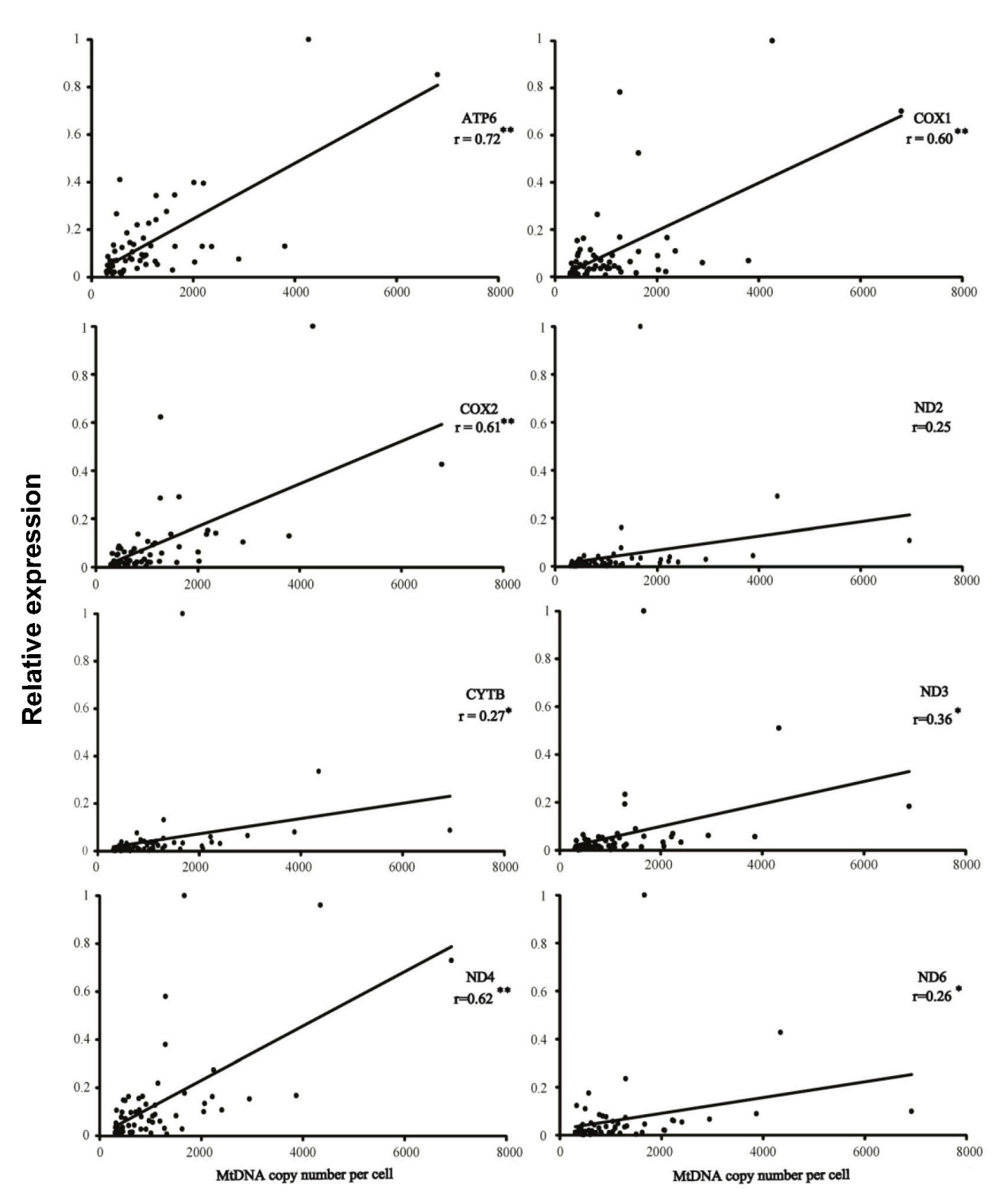

Figure 4. Linear regression analysis to establish the association between mitochondrial genes and mtDNA copy number for all samples $(* \mathrm{P}<0.05, * * \mathrm{P}<0.01)$.

In conclusion, we analyzed the mtDNA content in 8 tissues from pigs at 5 different stages of growth, from embryo to adult, using qPCR. We found that mtDNA content increased with growth for most tissues, and that this change may have been caused by the expression of TWINKLE. We also found that mtDNA copy number variation affected mitochondrial gene expression to regulate energy demands. Our results will provide basic data for investigations of tissue development, particularly muscle growth and adipose accumulation. 


\section{ACKNOWLEDGMENTS}

Research supported by grants from the National High Technology Research and Development Program of China ("863" Program, \#2013AA102502), the National Special Foundation for Transgenic Species of China (\#2014ZX0800950B and \#2011ZX08006-003), the Fund for Distinguished Young Scientists of Sichuan Province (\#2013JQ0013), and the Project of Provincial Twelfth Five Years' Animal Breeding of Sichuan Province (\#2011YZGG15).

\section{REFERENCES}

Bakala H, Ladouce R, Baraibar MA and Friguet B (2013). Differential expression and glycative damage affect specific mitochondrial proteins with aging in rat liver. Biochim. Biophys. Acta 1832: 2057-2067.

Barazzoni R, Short K and Nair KS (2000). Effects of aging on mitochondrial DNA copy number and cytochrome c oxidase gene expression in rat skeletal muscle, liver, and heart. J. Biol. Chem. 275: 3343-3347.

Cinti S (2006). The role of brown adipose tissue in human obesity. Nutr. Metab. Cardiovasc. Dis. 16: 569-574.

Clayton DA (1991). Replication and transcription of vertebrate mitochondrial DNA. Annu. Rev. Cell Biol. 7: 453-478.

Cypess AM, Lehman S, Williams G, Tal I, et al. (2009). Identification and importance of brown adipose tissue in adult humans. N. Engl. J. Med. 360: 1509-1517.

Das S, Ferlito M, Kent OA, Fox-Talbot K, et al. (2012). Nuclear miRNA regulates the mitochondrial genome in the heart. Circ. Res. 110: 1596-603.

Hoeks J and Schrauwen P (2012). Muscle mitochondria and insulin resistance: a human perspective. Trends Endocrinol. Metab. 23: 444-450.

Kaaman M, Sparks LM, van Harmelen V, Smith SR, et al. (2007). Strong association between mitochondrial DNA copy number and lipogenesis in human white adipose tissue. Diabetologia 50: 2526-2533.

Lanza IR, Short DK, Short KR, Raghavakaimal S, et al. (2008). Endurance exercise as a countermeasure for aging. Diabetes 57: 2933-2942.

Larsson NG and Gustafsson CM (2007). DNA replication and transcription in mammalian mitochondria. Annu. Rev. Biochem. 76: 679-699.

Latronico MVG and Condorelli G (2012). The might of microRNA in mitochondria. Circ. Res. 110: 1540-1542.

Masuyama M, Iida R, Takatsuka H, Yasuda T, et al. (2005). Quantitative change in mitochondrial DNA content in various mouse tissues during aging. Biochim. Biophys. Acta 1723: 302-308.

Milenkovic D, Matic S, Kühl I, Ruzzenente B, et al. (2013). TWINKLE is an essential mitochondrial helicase required for synthesis of nascent D-loop strands and complete mtDNA replication. Hum. Mol. Genet. 22: 1983-1993.

Miller F J, Rosenfeldt FL, Zhang C, Linnane AW, et al. (2003). Precise determination of mitochondrial DNA copy number in human skeletal and cardiac muscle by a PCR-based assay: lack of change of copy number with age. Nucleic Acids Res. 31: e61.

Moayyeri A, Hammond CJ, Hart DJ and Spector TD (2012). Effects of age on genetic influence on bone loss over 17 years in women: The Healthy Ageing Twin Study (HATS). J. Bone Miner. Res. 27: 2170-2178.

Pesce V, Cormio A, Fracasso F, Vecchiet J, et al. (2001). Age-related mitochondrial genotypic and phenotypic alterations in human skeletal muscle. Free Radic. Biol. Med. 30: 1223-1233.

Pohjoismäki JL, Goffart S, Taylor RW, Turnbull DM, et al. (2010). Developmental and pathological changes in the human cardiac muscle mitochondrial DNA organization, replication and copy number. PloS One 5: e10426.

Sripada L, Tomar D and Singh R (2012). Mitochondria: One of the destinations of miRNAs. Mitochondrion 12: 593-599.

Tyynismaa H, Sembongi H, Bokori-Brown M, Granycome C, et al. (2004). Twinkle helicase is essential for mtDNA maintenance and regulates mtDNA copy number. Hum. Mol. Genet. 13: 3219-3227.

Uddin M, Cinar M, Tesfaye D, Looft C, et al. (2011). Age-related changes in relative expression stability of commonly used housekeeping genes in selected porcine tissues. BMC Res. Notes 4: 441.

Wang J, Jiang J, Fu W, Jiang L, et al. (2012). A genome-wide detection of copy number variations using SNP genotyping arrays in swine. BMC Genomics 13: 273.

Welle S, Bhatt K and Thornton CA (2000). High-abundance mRNAs in human muscle: comparison between young and old. J. Appl. Physiol. 89: 297-304.

Yin P, Lee H, Chau G, Wu Y, et al. (2004). Alteration of the copy number and deletion of mitochondrial DNA in human hepatocellular carcinoma. Br. J. Cancer 90: 2390-2396. 